\section{Potential Use of Radiolabeled Antibodies for Imaging and Treatment of COVID-19}

TO THE EDITOR: I have read with interest the commentary by Pillarsetty et al. (1) in the December issue. Although the idea of investigating nuclear medicine-based solutions for treatment of coronavirus disease 2019 (COVID-19) is interesting and is potentially deserving of comment, the commentary by Pillarsetty et al. raises some concerns.

First, the only experimental data reported in the commentary are binding of the commercial antibody developed toward the spike protein of severe acute respiratory syndrome coronavirus 2 (SARSCoV-2) to that same commercial spike protein attached to magnetic beads. This piece of data, which is a simple quality control procedure for any antibody reagent received by a laboratory, does not prove the utility of detecting a virus such as SARS-CoV-2 in vivo.

Second, the authors did not use in their experiments a dead virus, a live virus, an attenuated strain, or a relevant in vivo model of COVID-19 infection, such as a ferret model (2). However, the title of their commentary promises not just one but several treatment options for COVID-19. The current situation, in which hundreds of thousands of COVID-19 patients are dying across the world, warrants proof of a therapeutic claim in at least one in vivo model of the disease.

Third, there is another issue with the title of the commentary. It states that the approach of targeting viral infections with radiolabeled antibodies for imaging or therapy is "oncology-inspired," when in fact at least 18 papers on targeting of viral infections for therapy with radiolabeled antibodies have been published since 2006 (3-7) and all of them have been listed on PubMed, presented at multiple nuclear medicine meetings and Department of Energy meetings, and covered by the press and Newsline. There have also been publications on radiolabeled-antibody imaging of viral infections in vivo in non-human primates $(8,9)$.

Finally, the commentary does not discuss the enormous radioresistance of virions and the implications of such radioresistance for suggested therapy targeting the virus directly. For example, 18 $\mathrm{kGy}$ of radiation are required to sterilize bone grafts from HIV virions (10), which belong to the same RNA virus type as SARS-CoV-2.

\section{DISCLOSURE}

Ekaterina Dadachova is a consultant and received research support from Actinium Pharmaceuticals and RadImmune Therapeutics. No other potential conflict of interest relevant to this letter was reported.

\section{REFERENCES}

1. Pillarsetty N, Carter LM, Lewis JS, Reiner T. Oncology-inspired treatment options for COVID-19. J Nucl Med. 2020;61:1720-1723.

2. Proud PC, Tsitoura D, Watson RJ. Prophylactic intranasal administration of a TLR $2 / 6$ agonist reduces upper respiratory tract viral shedding in a SARS-CoV-2 challenge ferret model. EBioMedicine. 2021;63:103153.

3. Dadachova E, Patel MC, Toussi S. Targeted killing of virally infected cells by radiolabeled antibodies to viral proteins. PLoS Med. 2006;3:e427.

(C) 2021 by the Society of Nuclear Medicine and Molecular Imaging
4. Casadevall A, Goldstein H, Dadachova E. Targeting host cells harbouring viruses with radiolabeled antibodies. Expert Opin Biol Ther. 2007;7:595-597.

5. Tsukrov D, McFarren A, Morgenstern A. Combination of antiretroviral drugs and radioimmunotherapy specifically kills infected cells from HIV-infected individuals. Front Med (Lausanne). 2016;3:41.

6. McFarren A, Lopez L, Williams DW. A fully human antibody to gp41 selectively eliminates HIV-infected cells that transmigrated across a model human blood brain barrier. AIDS. 2016;30:563-572.

7. Garg R, Mills K, Allen KJH. Comparison of various radioactive payloads for a human monoclonal antibody to glycoprotein 41 for elimination of HIV-infected cells. Nucl Med Biol. 2020;82-83:80-88.

8. Santangelo PJ, Rogers KA, Zurla C. Whole-body immunoPET reveals active SIV dynamics in viremic and antiretroviral therapy-treated macaques. Nat Methods. 2015; $12: 427-432$.

9. Dadachova E, Berman JW. Non-invasive nuclear imaging for localization of viral reservoirs. Nat Methods. 2015;12:399-400.

10. Grieb TA, Forng RY, Stafford RE. Effective use of optimized, high-dose (50 kGy) gamma irradiation for pathogen inactivation of human bone allografts. Biomaterials. 2005;26:2033-2042.

\author{
Ekaterina Dadachova \\ University of Saskatchewan \\ Saskatoon, S7N 5E5 \\ Saskatchewan, Canada \\ E-mail: ekaterina.dadachova@usask.ca
}

Published online May 7, 2021.

DOI: 10.2967/jnumed.120.261874

\section{Reply: Potential Use of Radiolabeled Antibodies for Imaging and Treatment of COVID-19}

REPLY: We are happy to respond to the 4 concerns raised by Dr. Ekaterina Dadachova in her letter to the editor commenting on our Brief Communication "Oncology-Inspired Treatment Options for COVID-19”'(1).

Regarding the first 2 points, we would like to emphasize that these experiments were performed during the height of the devastating first COVID-19 peak in New York City, when very little was known about the etiology, transmission, and possible treatments for this virus. Without the ability to use animal models, we nevertheless recognized that the uncertainty, the general dearth of knowledge, and the rapidly evolving situation at that time merited publication of our data as a Brief Communication to provoke thought and discussion on a potential approach to treating this disease.

Regarding the third point: the suggested references, including some interesting papers authored by Dr. Dadachova, were not appropriate for this Brief Communication because they do not address Auger therapy. Future publication of the ongoing studies may warrant citation of this work.

Lastly, the critical advantage of Auger therapy, as described in our Brief Communication, is that Auger electron cascades are highly localized, providing potential advantages over other forms of ionizing radiation when it comes to destroying virions. The letter's final point references the " $18 \mathrm{kGy}$ of radiation" required for sterilization of bone

(C) 2021 by the Society of Nuclear Medicine and Molecular Imaging 